\title{
Deadly Envenomation by Massive Bee Stings in a 7-Year Old Child in Ouagadougou
}

\author{
F. Ouédraogo ${ }^{1}$, C. Yonaba ${ }^{1}$, A. Kalmogho', C. Zoungrana ${ }^{1}$, \\ C. Bouda², O. Sawadogo'2, M. Delma², F. Koueta' ${ }^{1}$, L. Kam ${ }^{1}$
}

\author{
${ }^{1}$ Department of Pediatrics, Unit for Training and Research in Health Sciences (UFR/SDS), University Ouagadougou, \\ Ouagadougou, Burkina Faso \\ ${ }^{2}$ Department of Pediatrics, University Hospital Center Yalgado Ouédraogo (CHUYO) in Ouagadougou, Ouagadougou, Burkina \\ Faso \\ Email: *otraflore@yahoo.fr, caroyonaba@yahoo.fr, zangele2001@yahoo.fr, maiakaren@yahoo.fr, gcbouda@yahoo.fr, \\ marousaw@yahoo.fr, martidelma@yahoo.fr, kouetafla@yahoo.com, prkaml@yahoo.fr
}

How to cite this paper: Ouédraogo, F., Yonaba, C., Kalmogho, A., Zoungrana, C., Bouda, C., Sawadogo, O., Delma, M., Koueta, F. and Kam, L. (2017) Deadly Envenomation by Massive Bee Stings in a 7-Year Old Child in Ouagadougou. Open Journal of Pediatrics, 7, 289-293.

https://doi.org/10.4236/ojped.2017.74033

Received: September 8, 2017

Accepted: November 18, 2017

Published: November 21, 2017

Copyright $\odot 2017$ by authors and Scientific Research Publishing Inc. This work is licensed under the Creative Commons Attribution International License (CC BY 4.0).

http://creativecommons.org/licenses/by/4.0/

\begin{abstract}
Introduction: We are reporting a deadly case of massive bee stings observed in a 7-year-old child in Ouagadougou. Observation: The first symptoms, which appeared two hours after the stings of about 200 bees, motivated a consultation in a nearby dispensary in which the child received intravenous betamethasone before being referred to the University Hospital Center Yalgado Ouédraogo (CHUYO). Seven hours after the accident, a rapid deterioration in the general condition was noted with hemorrhage, respiratory distress, coma, and shock. A grade 3 anaphylaxis was diagnosed. The patient didn't survive despite of the cardiopulmonary resuscitation using adrenaline in intensive care. Conclusion: The massive bee stings have caused a severe biphasic anaphylactic reaction which could not be reversed by the adrenaline administered in the second phase. The development of a protocol would contribute to a better management of the cases.
\end{abstract}

\section{Keywords}

Envenomation, Massive, Stings, Bees, Ouagadougou

\section{Introduction}

The majority of insect stings are benign but some cases can be severe. We are reporting a deadly case by massive bees observed in the pediatric department of the University Hospital Center Yalgado Ouédraogo (CHUYO).

\section{Observation}

A 7-year-old girl, an unspecified paraplegic residing in Ouagadougou was received 
one day in December 2016 around $8 \mathrm{pm}$ for massive stings by bees that occurred at $4 \mathrm{pm}$. Two hours after the stings, bloody vomiting and coughing prompted consultation in a peripheral health facility during which the child received injectable betamethasone before being referred to the CHUYO.

On arrival the general condition was good, the weight at $15 \mathrm{~kg}$, an edema of the face was noted, as well as several hypochromic punctiform maculas (estimated to 200) localized on the head (Figure 1), neck, upper part of the trunk and limbs. Hospitalized, the patient presented starting from $11 \mathrm{pm}$, a rapidly progressive alteration of the general condition, fever, minor oral and nasal bleeding, respiratory distress, coma and a state of shock.

Blood counts showed a predominantly polynuclear hyperleucocytosis at 27,000/ $\mathrm{mm}^{3}$. The diagnosis of anaphylaxis grade 3 by massive envenomation due to bee stings was retained. Repeated adrenaline administration, oxygen therapy, vascular filling, injectable corticoids, oral and injectable antihistamines, and nebulized salbutamol did not reverse the state of shock. The Patient died around 8 am the following day, during the transfer to the intensive care unit of the University Hospital Center Charles De Gaule.

\section{Discussion}

Hymenoptera occupy the first place in terms of the risks of envenomation they expose humans to [1]. The phenomenon is underestimated due to the lack of notification. A single sting is the most common incident, but wasps, ants and bees can cause multiple stings. The African bee in particular is very aggressive and is involved in massive attacks in Africa but also in America where the Africanized

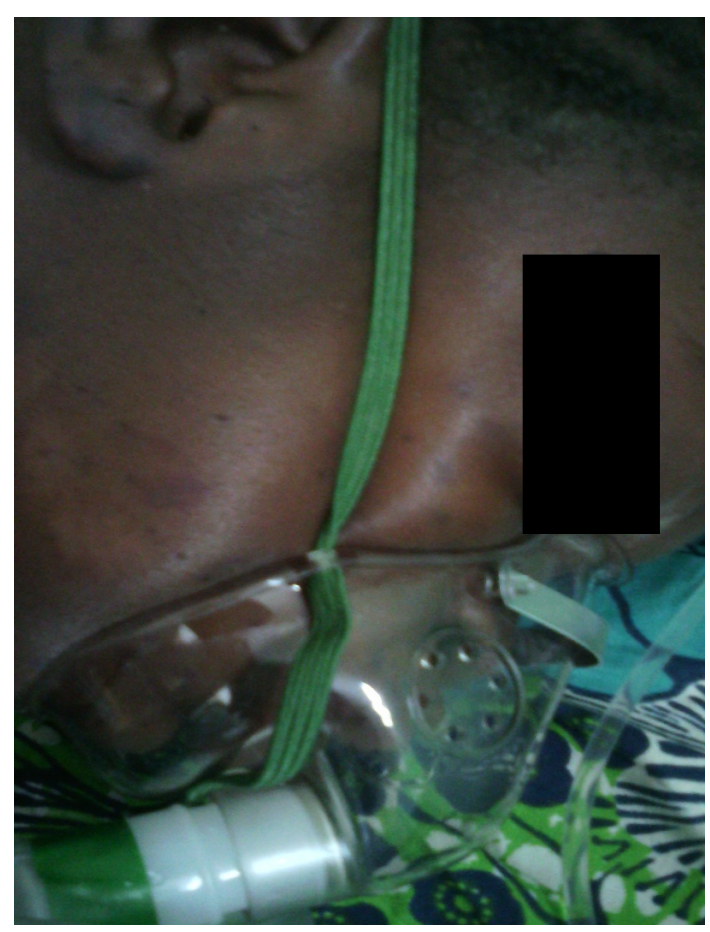

Figure 1. Patient under oxygen mask. 
bee obtained by the crossbreeding of the European bee and of the African bee [2] is rife.

The diagnosis of anaphylaxis was retained against the general manifestations that occurred after the bee stings. Systemic effect may be observed in case of prior sensitization to bee venom or following massive stings leading to the inoculation of a huge quantity of venom [2]. Apart from these circumstances, the reaction is generally a local or locoregional inflammation. Nevertheless, this reaction could be severe in case of cephalic localization of the stings due to obstructive angioedema of the airways. Delayed hypersensitivity is rarely observed. The notion of prior stings has not been reported for the patient, but the number of stings exceeds the threshold of one sting per kilogram that can lead to systemic effect in children [3].

The diagnosis of anaphylaxis was clinical in view of the combination of cutaneous, digestive, respiratory and cardiovascular symptoms that occur a few hours after exposure to a known allergen [4].

The interpretation of the kinetics of histamine or tryptase that are specific tests that may confirm anaphylaxis after exposure, are not commonly used and are not available in our context. The hyperleukocytosis observed is common in envenomed patients [2] and is explained by the inflammatory response.

The severity of anaphylaxis in the patient was specified by the Mueller classification, in 4 grades of increasing intensity, which is specific to the reactions induced by insect stings. Another classification, that of Sampson, includes 5 grades which do not depend on the triggering cause of anaphylaxis [4] [5].

The evolution of anaphylaxis was biphasic for this patient. After the first manifestations within the 2 hours after the stings, a "free interval" of about 5 hours preceded the following signs which were more severe and in line with what is reported by the literature. When anaphylaxis is progressively worsened, it is called a protracted form [4].

The physiopathology of anaphylaxis is incompletely elucidated. Clinical manifestations are due to the action of inoculated or released pro-inflammatory mediators, resulting in contraction of digestive smooth muscles, bronchoconstriction, mucus hypersecretion, airway oedema, vasodilatation and myocardial damage [4].

The effect of bee venom is due to its multiple components, comprised of more than twenty including histamine, melitin, apamine, hyaluronidase, phospholipases, serine protease protein, etc., having histamines liberator, vasodilator, hemolytic, neurotoxic and necrotic effects [2]. The consequences of massive stings, described during an autopsy, are cutaneous, muscular, renal, cardiac, hepatic cell necrosis [2]. The subsequent manifestations may be impossible to distinguish from anaphylaxis and are then called anaphylactoids. In practice, the term anaphylaxis may be used when the clinical symptomatology is suggestive [6].

The dosage of creatine phosphokinase, lactate dehydrogenase, aspartate aminotransferase, hemoglobinuria, myoglobinuria, uremia, serum creatinine can document the lesions due to massive inoculation of bee venom. These tests become 
abnormal only after a time period of 24 hours, and could not be carried out for our patient.

Treatment was aimed at tackling the immediate vital urgency that was anaphylaxis. The patient received adrenaline, the treatment of choice, which is used to block the cascade of mediators regardless of the pathophysiological mechanism [4]. However, the administration was not immediate, a situation often observed for anaphylaxis [5]. Prehospital administration is possible in developed countries with self-injectable syringes which are not available in our context. The possibility of losing up to $35 \%$ of the blood volume during the first ten minutes suggests using two large size venous ports for the filling of any anaphylactic patient [7]. Corticoids, antihistamines and salbutamol are second choice agents as a result of their slow and/or limited action on symptoms.

There is no bee anti-venom serum. The stings left by each bee after stinging were absent in the case of our patient. The means of their ablation is not specified, a razor is usually advised [4]. Since, the patient did not survive the shock, osmotic diuresis, peritoneal dialysis or hemodialysis, as well as alkalinization of the urine [2] could not be carried out.

Unlike what is reported in the literature in similar cases [2] [3] [8], no analgesic treatment was necessary, as no pain was reported for our paraplegic patient.

The patient died approximately 10 hours after the beginning of the second phase of anaphylaxis. The latter is known to be severe and life-threatening [4]. Massive bee stings can be deadly from 20 bites $/ \mathrm{kg}$ of weight [3] but cases of patients that have survived several hundred stings have been reported [2] [8] [9]. The scarcity of such accidents, the high number of stings, the biphasic form of anaphylaxis, the delay in providing care were detrimental factors to the survival of the patient.

\section{Conclusion}

Massive bee stings are rare. The biphasic anaphylaxis which occurred did not reverse after the administration of adrenaline in the second phase. The development of a protocol would contribute to a better management of cases.

\section{Conflict of Interest}

The authors do not declare any conflict of interest.

\section{Contribution of Authors}

All the authors contributed to the drafting of this document.

\section{References}

[1] Chippaux, J.-P. and Goyffon, M. (2006) Envenomations and Intoxications by Poisonous Animals in General. Medecine Tropicale, 66, 215-220.

[2] Almeida, R.A., Olivo, T.E., Mendes, R.P., et al. (2011) Africanized Honeybee Stings: How to Treat Them. Revista Da Sociedade Brasileira De Medicina Tropical, 44, 755-761. 
https://doi.org/10.1590/S0037-86822011000600020

[3] Silva, G.A., Pires, K.L., Soares, D.C., et al. (2013) RRH: Envenoming Syndrome Due to 200 Stings from Africanized Honeybees. The Revista do Instituto de Medicina Tropical de São Paulo, 55, 61-64. https://doi.org/10.1590/S0036-46652013000100011

[4] Gloaguen, A., Cesareo, E., Vaux, J., et al. (2016) Management of Anaphylaxis in Emergency Medicine. Recommendations of the French Society of Emergency Medicine (SFMU) in Partnership with the French Society of Allergy (SFA) and the Francophone Group of Resuscitation and Emergency Pediatrics (GFRUP), and the support of the Pediatric Society of Pneumology and of Allergology (SP2A). Annales Francaises de Medecine d'Urgence, 6, 342-364.

https://doi.org/10.1007/s13341-016-0668-2

[5] Athamaica, R.O., Soren, M., Carsten, B., et al. (2017) Pre-Hospital Treatment of Bee and Wasp Induced Anaphylactic Reactions: A Retrospective Study. Scandinavian Journal of Trauma, Resuscitation and Emergency Medicine, 25, 4.

https://doi.org/10.1186/s13049-016-0344-y

[6] Eigenmann, P., Köhli, A., Wassenberg, J., et al. (2009) Practical Aspects of the Diagnosis and Management of Anaphylaxis in Children. Paediatrica, 20, 4.

[7] Cheng, A. (2011) Emergency Treatment of Anaphylaxis in Infants and Children. Paedatr Child Health, 16, 41-46.

[8] Berdai, M.A., Labib, S., El Balbal, M. and Harandou, M. (2011) Bite by a Massive Swarm of Bees in a Child. Pan African Medical Journal, 10, 16.

[9] Akolly, D.A.E., Guedenon, K.M., Tsolenyanu, E., Bessi, L.K., Gnamey, D.K. and Atakouma, Y. (2016) Massive Envenomation by Bees Sting in a Child in Togo. Open Journal of Pediatrics, 6, 232-236. https://doi.org/10.4236/ojped.2016.63032 